\title{
Un diagnóstico de los procesos de planificación educativa en Costa Rica
}

\author{
Enrique Vilchez Quesada ${ }^{2}$ \\ Gaby Ulate Solis ${ }^{3}$
}

Resumen: la planificación en cualquier contexto, implica lograr una serie de objetivos que posibiliten superar o mejorar en alguna medida, una situación problemática determinada. Este trabajo busca exponer, cómo se ha desarrollado el proceso de planificación educativa en Costa Rica y los nuevos problemas en esta materia que tendrá que enfrentar el país en los próximos años.

Palabras claves: planificación, procesos, educación, desarrollo, migración.

Abstrac: the planning in any context, implies to achieve a series of objectives that you/they facilitate to overcome or to improve in some measure, a problematic certain situation. This work looks for to expose, how the process of educational planning has been developed in Costa Rica and the new problems in this matter that will have to face the country in next years.

Key words: planning, processes, education, development, migration.

Universidad Nacional. Escuela de Informática evilche: @una.ac.ir

Universidad Nacional. Disisión de Educología gsolis@una.ac.cr 


\section{Concepto de Planificación y Evaluación}

Antes de iniciar la discusión acerca de la planificación educativa en Costa Rica, es necesario aclarar lo que en el transcurso de este trabajo se entenderá por planificación y en particular por planificación educativa.

Planificar significa: "hacer explícita, de manera adecuada, la intención de nuestras acciones, mediante un proceso de previsión de necesidades y racionalización del empleo de los medios materiales y recursos humanos disponibles, con el propósito de alcanzar objetivos concretos en períodos de tiempo específicos" (Murillo, 1995: 27).

Siendo la planificación un proceso, no es sólo un conocimiento teórico sino también una técnica operativa, es decir, una serie de conocimientos, métodos, procedimientos, técnicas de investigación, organización y administración que tienen un efecto directo sobre las necesidades inmediatas ante una determinada situación.

Particularmente, la planificación educativa se entenderá como un proceso que se realiza tomando como base las siguientes etapas: diagnóstico, programación, ejecución y evaluación.

La etapa de diagnóstico tiene como finalidad develar las áreas más deficientes del sistema educativo en su conjunto, permitiendo determinar las prioridades de programación de acciones tanto preventivas como de mejoramiento.

La etapa de programación incluye uno de los aspectos más importantes de cualquier proceso de planeamiento: la formulación de los objetivos que se persiguen en un plazo establecido en el proyecto de planificación (estos en función de las necesidades educativas diagnosticadas). En esta etapa, también se concretan los recursos materiales y humanos con que se cuenta para el alcance de los objetivos y 
se elaboran los planes metódicos de acción en congruencia con las decisiones gubernamentales, para finalmente ser ejecutadas por los organismos administrativos responsables.

La última etapa de un ciclo de planificación, incluye la evaluación de dicho proceso. La evaluación consiste en un juicio objetivo y crítico de revisión permanente acerca de las decisiones y medios de acción involucrados en los proyectos de planificación educativa. Fundamentalmente abarca tres etapas:

- El estudio de los efectos: esta etapa se caracteriza por la observación de los impactos concretos que ha ocasionado el proceso de ejecución, en especial los efectos inesperados.

- El juicio de los objetivos: se refiere a la comparación entre los resultados obtenidos y los objetivos buscados.

- La innovación experimental: la idea de esta etapa consiste en generar las soluciones alternativas de las fallas del proceso.

La planificación educativa engloba dos aspectos en su programación. Por un lado, el sistema escolar en sí mismo, donde se abordan los problemas propios del sistema educativo, por ejemplo: la relación entre niveles, programas de estudio, tipos de evaluación, entre otros. Por otro, busca una integración con relación a las necesidades del desarrollo económico y social de acuerdo a las posibilidades del país en todos los ámbitos. Es por ello que la planificación educativa abarca como proceso dos dimensiones de diferentes escalas; una micro dimensión centralizada en las problemáticas directas del sistema educativo, y' una macro dimensión, relacionada con las políticas educativas nacionales que aspiran alcanzar un ideal de ciudadano y que, por tanto, en los procesos de planificación, deben tener una 
congruencia con el tipo de individuo que el país necesita. Una planificación educativa adecuada lleva implícita una estrecha relación entre ambas dimensiones.

Toda planificación educativa se identifica con el proceso de adopción de decisiones del gobierno a través de sus órganos legislativos y ejecutivos, es decir, es un proceso donde las alternativas y planes preparados por los técnicos se convierten en política. También es un proceso metódi$\mathrm{co}$, que necesita de organismos y personal especializado capaz de aplicar los resultados de la investigación y los métodos de análisis a la solución de los problemas del desarrollo educativo. Es destacable además, que en su etapa de ejecución, la planificación educativa depende en gran medida del aparato administrativo de la educación, quien es el responsable de programar el conjunto de actividades y estrategias para incorporar los cambios en las bases del sistema educativo. En conclusión, la planificación educativa es un proceso político, técnico y administrativo.

En las circunstancias actuales de un país subdesarrollado como el nuestro, la planificación exige un lugar privilegiado en todos los ámbitos. Por ser muchas las necesidades y escasos los recursos, se hace indispensable organizar acciones inteligentes para que con el menor desperdicio de tiempo y esfuerzos, se logren resultados eficaces y seguros.

Particularmente, en el campo educativo la planificación es el medio más viable para poder mejorar en alguna medida, los graves deterioros que ha sufrido su calidad como consecuencia de la crisis financiera de los últimos años. 


\section{Planificación Educativa en Costa Rica}

\subsection{Un Diagnóstico de la Crisis en el Sistema Educativo Costarricense}

\subsubsection{Ejemplos de la Crisis}

Una investigación realizada por tres importantes académicas de la universidad de Costa Rica durante el año de 1984; Zayra Méndez, Carmen Chávez y Ana Cecilia Escalante, sobre el desarrollo del pensamiento formal en los estudiantes de secundaria en el área metropolitana, reveló un estancamiento general en el desarrollo intelectual de los jóvenes, casi independientemente de su estrato social. Ello es una prueba tangible, acerca de la crisis educativa que ya desde la década de los ochentas se hacía presente en el sistema educativo en Costa Rica.

Se supone que el estudiante costarricense al concluir la primaria, debe estar en capacidad de demostrar dominio en matemática, español, estudios sociales y ciencias. En las pruebas de sexto grado de 1994 se eliminaron las notas de presentación de acuerdo a los promedios obtenidos durante cuarto, quinto y sexto año. Los porcentajes de reprobación nacionales, tomados del conjunto estudiantil, revelaron un fracaso de más de una cuarta parte de alumnos que perdieron al menos una materia, $24.8 \%$ en matemática, $19.5 \%$ en estudios sociales, $10.3 \%$ en ciencias y $8.2 \%$ en español ( $\mathrm{Gu}$ rriarán, 1994: 15).

Lo más representativo de estos datos cuantitativos, es que en 1994 las pruebas fueron diseñadas por los distritos escolares en que los estudiantes estaban matriculados, lo cual refleja un panorama bastante decadente en la calidad y cantidad de aprendizajes en los estudiantes de II ciclo.

Los resultados en las pruebas de bachillerato durante ese mismo año, revelaron porcentajes de pérdida de $35.21 \%$ 
en matemática, $12.72 \%$ en estudios sociales, $8.55 \%$ en ciencias, $9.19 \%$ en español y $7.76 \%$ en idiomas extranjeros (Gurriarán, 1994:15).

Durante el año de 1994 dos investigadoras Solís y Rodríguez pusieron en evidencia mediante datos cuantitativos de acuerdo con Gurriarán (1994: 16), que el 61\% de los estudiantes de undécimo año demostraron resultados de regulares a deficientes en la resolución de problemas y en lectura comprensiva. Estos resultados develaron el escaso rendimiento de la educación secundaria para preparar a los estudiantes a razonar y pensar crítica y creativamente.

Resultados tan alarmantes en el inicio de un nuevo gobierno en 1994, indudablemente impulsaron en Costa Rica la propuesta de planificación educativa denominada EDU-2005, que analizaremos posteriormente.

Con los ejemplos anteriores queda demostrado, de una forma tangible, la crisis educativa que Costa Rica arrastra desde hace muchos años, hasta nuestros días.

\subsubsection{Deficiencias del Sistema Educativo en Costa Rica}

Las razones de esta crisis tienen precedente en diversas fuentes.

\section{1) La crisis financiera}

La calidad de la educación ha sufrido graves deterioros principalmente como consecuencia de la crisis financiera que caracteriza a nuestro país. Las condiciones de trabajo del personal docente, cada vez peor remunerado, tienen efectos directos sobre su desempeño profesional. Así, en la mayoría de centros educativos, se presentan problemas de trastornos en la continuidad del proceso educativo con incapacidades frecuentes, ausentismos injustificados, pérdida 
de casi la mitad de tiempo de clase en asuntos no concernientes directamente con la enseñanza y el aprendizaje, entre otros.

En la actualidad, la falta de incentivos orilla al docente a estancarse en la apatía y el conformismo.

\section{2) La carencia de personal calificado}

Diversos estudios han demostrado la carencia de personal calificado tanto en primaria como en secundaria, principalmente en las regiones más alejadas de la capital en el territorio nacional.

\section{3) La pedagogía de los profesionales de la educación}

La pedagogía de los profesionales de la educación, se ha caracterizado por provenir de la llamada pedagogía científica desarrollada por algunos médicos y psicólogos bajo el estandarte del intelectualismo frívolo y de la figura autoritaria del maestro, esta última apoyada en buena medida por las raíces ético religiosas de nuestra cultura.

La formación de los docentes costarricenses se ha concentrado en la didáctica y la técnica. El pensamiento pedagógico en el país se ha orientado fundamentalmente bajo una mentalidad pragmatista de teorías extranjeras cambiantes, lo cual ha traído como consecuencia la falta de un conocimiento claro del pensamiento pedagógico custarricense. Podríamos considerar que dicho pensamiento es inexistente, sin embargo, la historia de nuestro país nos hace caer en la cuenta del desarrollo de un pensamiento educativo propio, fundado por ilustres educadores tales como: Florencio del Castillo, Mauro Fernández, Joaquín García Monje, Omar Dengo, Emma Gamboa, Constantino Láscaris, entre otros, que han moldeado en el transcurso del tiempo, 
una filosofía educativa remontada en nuestra cultura. Lamentablemente esta cultura sistemática se ha perdido en la formación de los docentes, por sustitución de las corrientes pedagógicas extranjeras, que a nuestro parecer, por ser foráneas, muchas veces son consideradas "modernas".

\section{4) Entusiasmo pasajero por métodos y técnicas pedagógicas}

Otra tendencia de nuestro sistema educativo, es el entusiasmo pasajero con que se promueven los métodos y técnicas pedagógicas contemporáneas. Lo criticable de esta tendencia es la poca conciencia con que se incorporan los cambios en el cuerpo educativo. En este sentido, los intereses políticos por lo general se imponen sobre el verdadero beneficio educativo, creando el estereotipo de considerar las corrientes pedagógicas contemporáneas por ser actuales, mejores para el país. Claro está, alrededor de este tipo de decisiones, imperan intereses políticos de los gobiernos, para atribuirse a sí mismos el fortalecimiento de un estado moderno en todos sus ámbitos. Es por ello que las políticas educativas en nuestro país son políticas de escritorio, impuestas a las domesticadas bases del sistema educativo. Lo grave de este tipo de medidas es que los docentes, en sus respectivas instituciones, acatan los cambios dispuestos en sus planeamientos, pero no en su práctica cotidiana, cayendo en un juego de engaños mediante el discurso, que afecta directamente la calidad de la educación.

5) Se centra la atención en el ¿cómo? y no en el ¿para qué?

Otra deficiencia de la enseñanza en las instituciones educativas, es que se centra la atención en responder al ¿cómo? y no al ¿para qué? del proceso educativo. Los docentes 
presentan una gran preocupación por saber cómo enseñarán los contenidos a sus estudiantes, lo cual ha tergiversado la enseñanza. Los maestros sienten que no deben abrumar al pobrecito estudiante exigiéndole una visión más profunda e integral del conocimiento, ya que éste requiere ir asimilando "cucharaditas" de conocimiento para alcanzarlo en su totalidad ¿Qué gran mediocridad!, la excesiva preocupación por la enseñanza, ha orillado a muchos docentes a creer que sus estudiantes son incapaces de entender el conocimiento de una forma más sistemática. El mismo Ministerio de Educación Pública (MEP), adopta esta política, al calificar a profesionales en ciencias puras como aspirantes en docencia, lo cual nos parece un grave error, pues estos profesionales tienen un manejo más profundo de la disciplina y por tanto pueden orientar mejor el ¿para qué? de su enseñanza. ¿Cómo puede resultar más importante para este ente administrativo por ejemplo, ser licenciado en la enseñanza de la química que premio Novel en química?, la comparación es exagerada pero una realidad.

\subsubsection{Efectos del Sistema Educativo en Costa Rica}

Los efectos del sistema educativo en Costa Rica nos podrían hacer pensar que la escuela es un fracaso muy costoso para el Estado. En la mayoría de los casos, la meta buscada por los estudiantes consiste en aprobar un examen o recitar mecánicamente una clase, provocando un aprendizaje nada duradero y el disgusto por el saber en los centros educativos. Este último aspecto, ha generado toda una estereotipación de la Escuela, considerada por la mayoría de los estudiantes desmotivante y aburrida. Lo anterior produce en su actitud, una repulsión automática hacia todo lo que se relaciona con la reflexión y el pensamiento, un ejemplo de ello es el desprecio que el alumno guarda por lo general hacia el estudio de la matemática. 
Todo esto ha provocado un grave problema de repitencia y deserción estudiantil en el país. En Costa Rica durante 1994, el índice de repitencia en primaria fue de $11.3 \%$ y el de deserción de 2.3\% (Gurriarán, 1994: 16). En secundaria estos problemas se agudizan, según las investigaciones de Rodríguez y Solís (1994) en cuanto a repitencia, sétimo ( $27.5 \%)$, noveno $(24.3 \%)$ y décimo $(31.9 \%)$ son los años de mayor reprobación casi independientemente de las materias cursadas por los estudiantes. El problema de la deserción en secundaria, según Rodríguez y Solís, inicia con el sétimo año donde más estudiantes abandonan sus estudios, seguido por la transición entre noveno y décimo. La deserción y fracaso estudiantil es un problema que atenta en contra del desarrollo en los países subdesarrollados.

El sistema educativo por defecto, en lugar de educar a los alumnos los escolariza con el fin de aceptar la sociedad tal y como les fue heredada. Los jóvenes estudiantes que se egresan de secundaria, claramente presentan graves deficiencias: una pésima ortografía, mal manejo de su idioma materno, un vocabulario sumamente pobre, una mala pronunciación, el hábito de lectura es incipiente, su lógica matemática es casi nula e integralmente es un individuo conformista y sin iniciativa.

Con mentes tan frágiles, su dominio se produce sin ninguna dificultad por la clase dominante. Es muy posible, que esta generación de analfabetas le resulte muy cara al Estado, pero al mismo tiempo muy conveniente para una élite selecta.

\section{El Ideal de Ciudadano que el Sistema Educativo debe Promover}

En el discurso, las características que el sistema educativo costarricense desea desarrollar en los estudiantes, 
supone la formación de: "ciudadanos capaces de pensar, que vean personas y no instrumentos en los demás, que busquen justicia, libertad, que se responsabilicen altruistamente de las consecuencias de sus acciones, que sepan pedirle más al esfuerzo propio que a las instituciones del estado, que amen a su patria y a sus semejantes como a sí mismos" (Doryan, $1995: 10$ ).

Las directrices del sistema educativo parecen no concurrir con los artículos sublimes de nuestra Ley Fundamental de Educación.

\section{Política Educativa EDU-2005}

La propuesta de educación para el año 2005 surge para asumir el reto de crear un sistema educativo de categoría mundial, frente a las exigencias racionalistas, humanistas, técnicas y ecológicas que el nuevo milenio demanda en la búsqueda de un desarrollo sostenible en todos los ámbitos.

En este contexto, el desarrollo del país estará en función de una educación que promueva:

- Sostenibilidad del recurso humano: bajo esta perspectiva la educación se convierte en un instrumento óptimo de movilidad social ascendente.

- Sostenibilidad ambiental: es necesario concienciar a los individuos de que sus acciones tienen repercusiones positivas o negativas sobre los recursos naturales y la biodiversidad.

- Sostenibilidad económica y productiva: la educación es el medio de producción en la formación de los recursos humanos capaces de elevar la competitividad internacional y la eficiencia del país.

- Sostenibilidad social y política: esta sostenibilidad depende de la formación de ciudadanos conscientes de sus 
derechos y deberes, capaces de asumir una conducta altruista con su comunidad y la sociedad en general.

La EDU-2005 representó la etapa de programación del proceso de planificación educativa en cuya continuidad, actualmente nos encontramos. Los graves problemas educativos evidenciados por diversas investigaciones principalmente las de Rodríguez y Solís durante el año de 1994, inyectaron la urgencia de un cambio en el sistema educativo, junto con las nuevas exigencias demandadas por la entrada del nuevo milenio. La Política Educativa hacia el siglo XXI fue un plan de planificación educativa que en estos momentos ha dado los insumos y resultados que están orientando el futuro de la educación costarricense.

\subsection{Los Objetivos de la Política Educativa Hacia el Siglo XXI}

La política educativa pretendió:

- Cerrar las brechas existentes entre la calidad de la educación que reciben los estudiantes de las áreas urbanas, rurales y urbanas marginales.

- Formar recursos humanos, que eleven la competitividad en los mercados internacionales.

- Fortalecer valores que se han ido perdiendo con el pasar del tiempo.

- Estimular el respeto por la diversidad cultural, social y étnica.

- Hacer conciencia en los individuos acerca de la necesidad de un desarrollo sostenible económico y social en armonía con la naturaleza.

- Fortalecer la educación técnica y científica a la par de la deportiva y la cultural, como forma de estimular un desarrollo integral. 
El tema del desarrollo sostenible se hace presente en esta política educativa, como representante de una nueva exigencia para alcanzar un verdadero desarrollo en el presente siglo. La educación bajo esta perspectiva, se convierte en el instrumento más eficaz para incorporar en la idiosincrasia costarricense, la necesidad de velar por una relación equilibrada con la naturaleza y el entorno en general, con todo lo que esto conlleva; cambiar nuestros hábitos de consumo, no adquiriendo productos por ejemplo que atenten contra la capa de ozono; cambiar nuestra propia actitud con lo que respecta al aumento de la contaminación, evitando botar basura en las calles, entre otras medidas.

Fue en su momento un nuevo planteamiento en una política educativa, que buscaba no comprometer a las futuras generaciones.

\subsection{Fuentes Filosóficas}

La Política Educativa actual se centra en tres corrienres filosóficas básicas:

\section{El humanismo}

Se quiere crear individuos con solidaridad y una visión de largo plazo, que realicen el deseo de dejar un mundo mejor para las futuras generaciones, crear individuos con dignidad humana y hábitos saludables que beneficien su desarrollo.

Con relación a este último aspecto, es muy importante que se enseñe a los estudiantes hábitos alimenticios. En los centros educativos, se ven reflejados los hábitos tan desastrosos que los estudiantes tienen en su alimentación. Sería interesante investigar, si existe alguna relación entre los hábitos alimenticios de los alumnos y su rendimiento académico. 


\section{El racionalismo}

Procura que los participantes desarrollen el razonamiento y la facultad de conectar las ideas, consciente, coherente e intencionalmente.

Desde este punto de vista, la educación debería formar individuos capaces de pensar inteligentemente por sí mismos, sin embargo, es claro que el papel pasivo de los estudiantes se sigue manteniendo con mayor predominio en los centros educativos.

\section{El constructivismo}

Plantea la necesidad de desembocar en el salón de clase en una autonomía que posibilite a cada participante construir su propio conocimiento. Esta autonomía puede dividirse en tres: la intelectual, la moral y la socio-afectiva.

Esta corriente pedagógica podría catalogarse actualmente como contemporánea, esperemos que el apasionamiento que el Ministerio de Educación Pública ha mostrado por ella, no se apague cuando deje de estar en boga. Lo cual es una característica común de las reformas educativas en el país, impulsar lo moderno y desechar las corrientes pedagógicas pasadas. Esta tendencia, ha traído graves trastornos en el sistema educativo costarricense, que sufre cambios relativos cada cuatro años. La educación requiere políticas educativas más estables. No podemos sacrificar el orden institucional con reformas pasajeras que no tienen ningún efecto positivo real en las bases de la educación.

\section{La Práctica de la Política Educativa}

Lo práctico de la Política Educativa se centra en seis aspectos: 


\section{Planes y programas}

Constituyen el currículo que sirve como guía a los profesionales de la educación, padres de familia y estudiantes.

En el diseño curricular propuesto por el Ministerio de Educación Pública se cumplen dos objetivos primordiales; el primero garantizar que independientemente de la ubicación geográfica del centro educativo, se establezca una uniformidad en el aprendizaje y construcción de los conocimientos, para que por igual todos los alumnos tengan las mismas oportunidades y se inserten exitosamente en el siglo XXI.

En nuestra opinión, nos parece que la educación costarricense no debería ser uniforme en las distintas comunidades, ya que cada una presenta deficiencias y dificultades de diferente índole. Unificar la educación significa ignorar la individualidad de cada comunidad. Por ejemplo, en una comunidad como La Carpio en San José ${ }^{4}$, ien qué medida es efectiva la enseñanza de la computación a los niños?, la mayor parte de los jóvenes que se egresan de la primaria no valoran la posibilidad de ingresar a la educación secundaria, en primer lugar por la urgencia de generar ingresos económicos en el hogar y en segundo lugar, no encuentran ningún sentido en la educación en general. La realidad es que este tipo de comunidades por sus altos índices de deserción, requieren programas de enseñanza orientados al aprendizaje de ciertos oficios. Ficticiamente se considera que por la entrada del nuevo siglo, la informática es insustituible en el sistema educativo, sin embargo, poblaciones como La Carpio donde las familias viven en ranchos bajo condiciones de pobreza extrema, ¿qué sentido podrá tener una computadora?,

4 En Costa Rica esta zona urbano marginal, se caracteriza por tener una condición socioeconómica muy baja y poseer altos índices de violencia y problemas sociales de diversa índole. 
estas poblaciones requieren, además, un aprendizaje de oficios, para optar por trabajos honrados que den un aporte positivo a la sociedad.

El segundo objetivo del diseño curricular, consiste en estimular el desarrollo cognoscitivo de inteligencias múltiples; inteligencia lingüística; artística y lógico matemática.

El currículo nacional básico, incluye también dentro de su estructura, la reflexión, discusión y práctica sobre valores morales, éticos y espirituales en todas las materias de los distintos ciclos y niveles.

Formación, capacitación y educación continua del educador

Se desea impulsar un aumento en los porcentajes de docentes calificados profesionalmente (es conocida la gran cantidad de docentes que no cuentan con un título universitario), además se pretende desarrollar la capacidad de los maestros y profesores para hacer frente a los retos que la Política Educativa plantea (por ejemplo el constructivismo).

\section{Evaluación}

Se pretende impulsar la práctica de las dimensiones diagnóstica y formativa de la evaluación. La evaluación cumple tres funciones: una diagnóstica, una formativa y una sumativa. La primera permite determinar el estado actual del proceso, la segunda posibilita una retroalimentación para efectos de ajustar las áreas más problemáticas y la tercera se caracteriza por utilizar datos cuantitativos para valorar el producto final.

\section{Recursos didácticos}

El Ministerio de Educación Pública plantea que los recursos didácticos deben jugar un papel relevante en el 
proceso de construcción del conocimiento. Este es un aspecto contradictorio de la política, ya que en la mayoría de centros educativos los únicos recursos disponibles para el docente son la tiza y el borrador.

\section{La mediación del educador}

La mediación del educador se define didácticamente como la interacción entre el aprendiz y un experto, en el contexto de la Política Educativa actual la mediación debe estar fundamentada en procesos constructivistas, en un ambiente de aprendizaje heurístico donde se desarrollen habilidades cognoscitivas y de pensamiento.

\section{Intermediación gerencial}

Los procesos administrativos característicos del Ministerio de Educación Pública, implican en la mayoría de los casos desmotivación por parte de los docentes y padres de familia, que no reciben el trato que se merecen. Uno de los objetivos de la Política Educativa, ha sido agilizar la capacidad administrativa del Ministerio y a su vez modernizar aquellos procesos que lo requieran.

En este sentido, las acciones concretas que el Ministerio se propuso en la EDU-2005 fueron las siguientes:

- Para 1998 reducir en 20 minutos el tiempo promedio para ser atendido en cualquier parte del Ministerio. Para el año 1999 , reducir a 15 minutos el tiempo para ser atendido y llevarlo a 10 minutos o menos en el año 2005.

- Para 1997, reducir a menos del 3\% la cantidad de educadores con trámites pendientes en procedimientos legales y llevar esa cantidad a un $0 \%$. 
- Para 1996 reducir a menos del 10\% la cantidad de docentes que no recibían pago en el mes de marzo y llevar esa cantidad a un $5 \%$ para marzo de 1998 y $1 \%$ para marzo de 1999.

- Establecimiento de un perfil de contratación de docentes y oficinas regionales para la selección de docentes (lo cual ya es una realidad).

- Simplificar y eliminar requisitos innecesarios en los procesos administrativos.

- Para 1998 reducir el número de interinos a 20\%, $15 \%$ para el $2000,10 \%$ en el 2002 y menos del $5 \%$ en el 2005 ( sin embargo, la oferta de propiedades del Ministerio de Educación es mínima, por ejemplo en el año 1999 en el área de la enseñanza de la matemática para todo el país, se ofrecieron ocho propiedades en zonas bastante alejadas).

- Capacitación del personal administrativo en comunicación, servicio al cliente y control estadístico de calidad.

Es muy preocupante observar que todavía en este año 2010 pocas de estas acciones son una realidad.

\section{Programas de Mejoramiento Impulsados por el MEP}

\section{El Bachillerato Internacional y el Programa de Años Medios}

El programa de bachillerato internacional surgió a mediados de los años sesenta en Ginebra (Suiza) como un medio de nuevas oportunidades para los estudiantes entre los 16 y 18 años de edad, mediante un programa de estudios de excelencia que incrementara sus posibilidades de ingreso a las universidades más altamente calificadas, independientemente de su país de origen. 
En la actualidad se ofrece a 75 países en 600 centros educativos alrededor del mundo. A partir del año 1995 la organización Bachillerato Internacional ha experimentado con un nuevo proyecto, el Programa de Años Medios dirigido a jóvenes entre los 11 años y 16 años de edad.

Ambos programas tienen en común la formación de actitudes y valores dentro de opciones curriculares en donde lo formativo no está separado de la construcción del conocimiento. Algunas de sus características son las siguientes:

- Su propósito es despertar las inteligencias de los estudiantes y enseñarles a relacionar los contenidos de clase con la realidad del mundo.

- El currículo interdisciplinario combina las ciencias sociales y naturales con los idiomas y la matemática.

- La comunicación ocupa un lugar preponderante, el estudiante aprende a tener un claro dominio de su lengua materna.

- Se pone énfasis en el aprendizaje de otros idiomas, con ello el estudiante enriquece su propia cultura y aprecia la idiosincrasia y visiones de mundo de otros.

- La investigación es parte integral de la labor del estudiante. En el Bachillerato Internacional el alumno debe completar un curso de teoría del conocimiento y escribir una monografía (estudio sobre un tema específico) de 4000 palabras. En el Programa de Años Medios el estudiante debe completar un proyecto en el que se reflejen sus conocimientos y su capacidad para resolver problemas.

- Las horas lectivas son de 60 minutos distribuidos en una mayor jornada diaria escolar.

Estos programas indudablemente egresan individuos con una amplia visión de mundo y capaces de pensar por sí 
mismos, sin embargo, el acceso a este sistema educativo es totalmente elitista, asignado únicamente a los hijos de las familias más poderosas económicamente en el país.

Con el Programa de Años Medios y el Bachillerato Internacional, se buscó mejorar la situación de la educación en proyección al año 2005.

\section{Incremento del año lectivo}

El incremento del año lectivo es actualmente una realidad. El curso lectivo se incrementó a 210 días, con una efectividad real de 200 días.

El Ministerio de Educación Pública también se propuso en 1995, aumentar la hora lectiva de 40 minutos a 60 minutos para el año de 1999. Es claro que este objetivo aún no se ha concretado. También tomó el reto de aumentar el día escolar a 6 horas. En la actualidad dudamos mucho que el MEP pueda conseguir esta meta, por las implicaciones de infraestructura y de personal que exigiría. Muchos colegios tienen una jornada diaria bastante comprometida, al funcionar como colegios diurnos y nocturnos al mismo tiempo, si a esto le sumamos el aumento de la población estudiantil en las instituciones, esta meta se convierte en casi una utopía.

\section{Programa de incentivos para el educador costarricense}

Al mejorar las condiciones laborales y la calidad de vida de los docentes, indirectamente se producen efectos positivos sobre su trabajo profesional. De allí que el MEP proponga:

- A partir de 1998, en aquellos centros que demuestren 
excelencia en el trabajo de equipo mediante el resultado de las pruebas nacionales, bajos índices de deserción, repitencia y ausentismo de docentes, además de proyectos de mejoramiento educativo, se incrementará entre un cuarto de salario a un salario extra a cada profesional con dos años en la institución. No cumplido.

- A partir de 1997, un salario extra por la permanencia en las zonas de menor desarrollo socioeconómico a profesionales con título de bachiller o mayor. Cumplido.

- A partir de 1997, modificación del sistema de pago de novenos por uno de doceavos, establecido el calendario escolar de 180 días efectivos. Cumplido.

- A partir de 1998, incentivo para los docentes que cumplan con la asistencia y puntualidad durante todo el curso lectivo de 180 días. Se pagará en el mes de enero un $50 \%$ extra sobre la base salarial, o bien entre 175-179 días laborados incentivo de un 30\%, entre 174-170 días, incentivo del 10\% del salario base. No cumplido.

- A partir de 1997, incentivo salarial para los profesionales de la educación especial, de un $40 \%$ sobre la base salarial. Cumplido.

- A partir de 1998, incentivo a un grado de ascenso a los profesionales que ocupan cargos de dirección y a los docentes de los centros educativos que dirigen, si cumplen con los indicadores de calidad del MEP. Cumplido.

Queda aún mucho qué mejorar. 


\section{Programa Tarea Nacional de Valores}

Este programa es una respuesta ante la preocupación en el país, por la pérdida de valores y continuos ejemplos negativos que en los últimos años la sociedad costarricense ha expuesto a las nuevas generaciones. El programa devuelve a la educación su rol protagónico en la formación integral del ciudadano.

El programa Tarea Nacional de Valores contempla dos etapas. La primera consiste en clarificar los valores y las actitudes que la familia y la comunidad, tanto nacional como la particular, desean cultivar y promover.

Con respecto a los valores y actitudes de la comunidad en particular, estás fueron propuestas por cada centro educativo mediante una declaratoria llamada: Declaratoria de Compromiso con una Cultura de Calidad Institucional, remitida a la dirección regional correspondiente, durante el mes de abril de 1997. Dicha declaratoria incluye además, la política de eficacia administrativa que rige a la institución, las estrategias de supervisión con respecto al trabajo profesional; las estrategias para el uso eficaz y efectivo de los materiales didácticos, los centros de recursos (bibliotecas) e instalaciones en general. Se supone que la evaluación de los resultados de dicha declaratoria, se realizó en el año 2000.

La segunda etapa, consiste en implementar cada año tres grades tareas de reflexión sobre valores y actitudes, que involucren a todos los estudiantes y profesionales de la educación, como también a las familias y comunidades. Cada centro educativo, debe elaborar estrategias de seguimiento y evaluación anual, con respecto al nivel de logro alcanzado. 


\section{Programa de proyectos de mejoramiento educativo}

Este programa consiste en financiar cada dos años un proyecto de mejora curricular, por el cual compiten los centros educativos que así lo deseen. Inició en el mes de setiembre de 1997 bajo la competitividad de 50 instituciones de secundaria y 500 de primaria.

Los docentes del centro educativo ganador con dos años de servicio en la misma institución, recibieron un reconocimiento salarial entre un cuarto de salario a un salario extra en junio de cada año de vigencia del proyecto. Además el Ministerio de Educación se comprometió a financiar toda publicación de materiales, que se generen como producto de la experiencia y divulgar ampliamente los resultados en los medios de comunicación.

En el año de 1999, finalizó el primer proyecto de mejoramiento educativo impulsado por el MEP. Se pretendió que este programa fuera de carácter permanente.

\section{Programa de planificación y evaluación del clima organi- zacional del centro educativo}

Se entiende por clima organizacional el conjunto de factores como normas, valores, actitudes y creencias compartidas por los miembros de una organización. En este sentido, la dirección juega un papel vital para construir un espíritu compartido de logro, en el cual se complemente la necesidad de hacer el trabajo con un alto nivel moral en el equipo.

El programa otorgó un incentivo económico equivalente a un grado de ascenso en la escala salarial a aquellos directores (as) que demostraron una tarea de coordinación que optimizó la calidad del clima organizacional del centro educativo a su cargo. 
Este programa inició en el mes de setiembre de 1997, paralelo al Programa de Proyectos de Mejoramiento Educativo.

\section{Nuevos Problemas en Materia de Planificación Educa- tiva en Costa Rica}

\subsection{Planificación e Inmigración}

La inmigración sobre un país, tiene efectos directos sobre aspectos tales como: la fuerza de trabajo, empleo y desempleo, la productividad, la salud, la educación, entre otros. En nuestro país, este fenómeno se ha incrementado a partir del año de 1992 y las consecuencias de un inadecuado planeamiento nacional, actualmente se hacen sentir.

En su mayoría, la población inmigrante en el territorio, la constituye las familias nicaragüenses que han emigrado de su país natal en la búsqueda de mejores oportunidades. Por esta razón, nos centraremos principalmente en el fenómeno migratorio nicaragüense.

\subsection{La Emigración Nicaragüense}

Las corrientes más importantes de emigraciones nicaragüenses confluyen como destinos finales en Costa Rica y Estados Unidos y quizás con menor representatividad Honduras y Guatemala usados como países trampolines. El volumen de nicaragüenses hacia Costa Rica supera ligeramente a los Estados Unidos (Brenes, 1999).

A través de las dos últimas décadas, la emigración nicaragüense puso énfasis en tres períodos con relativa importancia a estos dos países de destino. Estos tres períodos los constituyen: antes de 1990, de 1990 a 1992 y posterior a 1992. Durante el primer período, el país elegido por la mayoría 
de los emigrantes fue Estados Unidos intensificándose desde 1985 hasta 1988. A partir de los años 90, 91 y 92 , debido a los cambios políticos que se iniciaban en Nicaragua con un nuevo gobierno bajo perspectivas de un mejoramiento en la calidad de vida de sus habitantes, se propició un retorno de gran cantidad de ciudadanos a su país. Posteriormente al no cumplirse las expectativas creadas, se inicia una fuerte incursión de nicaragüenses al territorio costarricense, mientras que la emigración a Estados Unidos disminuye (Brenes, 1999).

\subsection{Escolaridad de los Inmigrantes Nicaragüenses}

Uno de los factores que influyen en los emigrantes para tomar la decisión de abandonar su país natal; es su nivel de escolaridad alcanzado. La población emigrante más calificada es la que posee mayores alternativas de empleo y es la que fácilmente se adapta a los nuevos patrones culturales, laborales y éticos.

En la conferencia Nicaragüenses en el Exterior, dictada durante el seminario internacional La Población del Istmo al Fin del Milenio en 1999 por José Rosales, se evidenció que los inmigrantes nicaragüenses con destino a Estados Unidos, poseen más educación que los de Costa Rica, esto se debe explicó, a las características de cada país y a las actividades que los inmigrantes pretenden realizar en el país de destino. En el nuestro, las expectativas giran en tomo a actividades agropecuarias, servicios hoteleros, restaurantes, construcción, servicio doméstico y en general labores mayoritariamente de gran esfuerzo físico y en condiciones no óptimas. 


\subsection{Planificación en el Área Educativa}

La cantidad de nicaragüenses legales que residen actualmente en nuestro país, se estima hasta el 31 de julio de 1999, según la investigadora Stella Chinchilla Mora de 275 465 , a este número hay que sumarle aquellos nicaragüenses en calidad de ilegales en el territorio. La Dirección Nacional de Migración y Extranjería no posee los recursos para estimar de forma factible el número de nicaragüenses que realmente viven en el país.

El estadístico Gilbert Brenes en su trabajo de tesis Estimación del Volumen y Características Sociodemográficas de los Inmigrantes Nicaragüenses en Costa Rica, aplicó métodos indirectos con el objetivo de ponderar la cantidad de nicaragüenses durante el año de 1999 en el territorio nacional, él establece un intervalo entre 320000 y 360000 inmigrantes.

En 1998 había en Costa Rica 17643 estudiantes nicaragüenses registrados por el Ministerio de Educación Pública, una cifra elevadísima en comparación con otros países centroamericanos. La falta de planificación y control de este ente administrativo, sugiere la posibilidad de que esta cifra sea muchísimo mayor.

Cualquier extranjero que desee ingresar al sistema educativo costarricense, puede hacerlo de inmediato, presentando los documentos migratorios legales y las notas de calificaciones certificadas en los países de origen, sin embargo, estos requisitos exigidos por el Ministerio de Educación Pública no son tan necesarios. Costa Rica posee un convenio internacional entre el PANI y la UNICEF, que protege el derecho al estudio de cualquier extranjero. En términos de este acuerdo un niño o joven indocumentado tiene todo el derecho a recibir su educación general básica. Los centros educativos y las direcciones regionales parecen no conocer este acuerdo. 
De cualquier forma, el ingreso al sistema educativo costarricense es relativamente fácil para los niños y jóvenes extranjeros y en particular los nicaragüenses. El Ministerio de Educación Pública, no ha tomado todavía medidas de planificación educativa, ante la masa de estudiantes nicaragüenses que en la actualidad se encuentran en las instituciones.

Dada la enorme cantidad de estudiantes en estas circunstancias, ignorar la situación no es la decisión más inteligente. Dentro de la planificación anual de la futura matrícula escolar este rubro es inexistente ya que el Ministerio no ha podido estimar el número de jóvenes y niños nicaragüenses que viven en Costa Rica.

Este reto en materia de planificación educativa es una necesidad para no poner en riesgo la educación costarricense y propone diversos dilemas, tales como:

- ¿El nivel de instrucción de los jóvenes nicaragüenses inmigrantes será menor que el de los jóvenes costarricenses?

- Si la mayor parte de los nicaragüenses que ingresan al país no cuentan con una educación general básica; ¿será necesario incorporar programas de educación para adultos?, o ¿sirve más al país mantener esta mano de obra barata?

- Si la Dirección Nacional de Migración y Extranjería no cuenta con los recursos para establecer una estimación de inmigrantes en el país; ¿cómo podrá llevar a cabo esta tarea el Ministerio de Educación Pública?

- Dado que la cultura de los jóvenes nicaragüenses es otra: ¿será necesario implementar nuevos tipos de escuela y programas de estudio que respeten su idiosincrasia?

La polémica es muy grande y difusa, sin embargo, lo que sí es claro, es la importancia de una planificación educativa frente al fenómeno migratorio en Costa Rica. 


\section{Conclusiones}

Los procesos de planificación educativa en Costa Rica se han impulsado desde la década de los noventas como un medio para mejorar la calidad del sistema educativo costarricense, que desde la década de los ochentas, viene dando claros indicios de inconsistencias en la formación general básica de los ciudadanos. Ante este panorama, emergió la política educativa EDU-2005 que formó las bases administrativas, filosóficas, pragmáticas y epistemológicas de la política educativa actual en Costa Rica.

Los retos a enfrentar en materia de planificación educativa en nuestro país son múltiples y diversos, sin embargo, en los próximos años particularmente el problema migratorio de la población nicaragüense que reside en Costa Rica, es uno de los mayores retos a enfrentar en las instituciones de enseñanza; ignorar el impacto de este fenómeno irá en perjuicio de la calidad del sistema educativo en general.

\section{Referencias bibliográficas}

Arias, R. (1994). Desafíos de la planificación educativa. Costa Rica: División de Planeamiento y Desarrollo Educativo (Servicio de documentación del departamento de planes y programas del MEP).

Bernardini, A. (1984). Reflexiones sobre el modelo educativo costarricense. Revista Tiempo Actual, 33, 19-32.

Brenes, G. (1999). Estimación del Volumen y Caracteristicas Sociodemográficas de los Inmigrantes Nicaragüenses en Costa Rica. Tesis de maestria. Universidad de Costa Rica.

Chinchilla, S. (1999). El impacto de la migración nicaragüense en el sistema educativo de Costa Rica. Costa Rica, Seminario Internacional: La Población del Istmo al Fin del Milenio. 
Doryan, E. (1995). Una propuesta de un proyecto educativo nacional hacia el año 2005. Costa Rica: Ministerio de Educación Pública.

Doryan, E. (1995). Marco conceptual de la politica educativa EDU2005. [intemet], http: //www.mep.go.cr /educación/ politica, asp.

Gurriarán, J. (1994). La planificación educativa en el futuro. Costa Rica: División de Planeamiento y Desarrollo Educativo (Servicio de documentación del departamento de planes y programas del MEP).

Maya, A. (1997). Ideas y elementos básicos para el planeamiento didáctico permanente de la práctica docente en el aula, San José, C.R. Editorial EDITORAMA.

Méndez, C. Educación y desarrollo: una relación dialéctica. Costa Rica: Universidad Nacional (Centro de investigación y docencia en educación).

Murillo, C. (1995). Ensayos sobre desarrollo. Heredia, C.R. Editorial EFUNA.

Rodríguez, G. (1975). La autogestión pedagógica. Costa Rica (CE. DAL, seminarios y documentos).

Rosales, J. (1999). Nicaragüenses en el exterior. Costa Rica, Seminario Internacional: La Población del Istmo al Fin del Milenio.

Trinidad, A., Pérez, M. (1998). Evaluación de programas de intervención social. Revista del Ministerio de Trabajo y Asuntos Sociales de Madrid, 113-119.

Vílchez, E. (1999). [Entrevista con la Lic. Marta Mora, asesora nacional del MEP]. 